\title{
BIO.07 - Anti-Acinetobacter baumannii monoclonal antibody as a potential immunological tool
}

Anna Erika Vieira de Araujo ${ }^{1 *}$; Milena Mouta Verdan França Carvalho ${ }^{1}$; Fernando de Paiva Conte ${ }^{1}$; Marcia Arissawa ${ }^{1}$; Ana Paula D’Alincourt Carvalho-Assef²; Jose Procopio Moreno Senna ${ }^{1}$.

1Fiocruz/Bio-Manguinhos;

2Fiocruz/IOC.

Introduction: Acinetobacter baumannii is an important opportunistic pathogen, with high incidence in intensive care units, mainly affecting immunocompromised patients. The increasing resistance to $\beta$-lactam antibiotics, especially to carbapenems, complicates the treatment and raises the need for novel therapy approaches, such as immunotherapies. Development of monoclonal antibodies (mAbs) against multi-drug resistant bacteria is well established in the literature. However, their true potential has not been fully tapped. In general, mAbs present high pathogen specificity, favorable pharmacokinetics with great versatility, allowing their use to not only immunotherapies but also in immunodiagnostics.

Objective: Regarding the multi-drug resistance problematic, this study aims to develop an anti-A. baumannii monoclonal antibody.

Methodology: First, three female BALB/c mice were immunized intraperitoneally with $25 \mu \mathrm{g}$ of a recombinant $A$. baumannii protein and Freund's adjuvant every 2 weeks. Mouse splenocytes were then isolated after the fourth immunization, fused with SP2/0 murine myeloma cells to obtain hybridomas and ELISA was used for screening. Briefly, $5 \mu \mathrm{g} / \mathrm{mL}$ of recombinant protein in $50 \mathrm{mM}$ carbonate-bicarbonate buffer was used as the coating agent, followed by incubation with hybridoma supernatants. Preimmune mice sera were used as negative controls, and binding of antibodies to the recombinant protein was detected by goat anti-mouse IgG-peroxidase conjugates. TMB was used as substrate and reading was performed with absorbance at 450nm. Hybridomas that produced positively bound antibodies were cloned with limiting dilution and grown in DMEM FBS 20\%/HT medium. Isotypes of the mAbs were determined using Pierce ${ }^{\circledast}$ rapid isotyping kits and western blot assays demonstrated $\mathrm{mAb}$ recognition to A. baumannii proteins.

Results: Only one of the three animals generated stable antibody-producing hybridomas. From these cells, the ones with ELISA absorbance values higher than 2.0 were selected for cloning. Hence, 9 polyclonal hybridomas were cloned, where six were recloned in a way to guarantee their stability. All presented themselves as IgG1 subtype, except one (NG4) that had IgG2a isotype. In the western blot assays, it was observed antibody recognition for the recombinant protein in all, except one, of the tested supernatants. In contrast, eight supernatants were tested against $A$. baumannii lysate proteins. From these, two showed no recognition, while four presented a discrete binding to one protein in the bacterial lysate, and two demonstrated excellent antigenic recognition to a single protein of $A$. baumannii.

Conclusion: Data together demonstrate that it was possible to obtain antibody-producing hibridomas that recognizes the target protein of $A$. baumannii, whether in the recombinant or the native form. These antibodies may have a potential use in therapy or diagnostics, but it all depends on how the antibody interacts with its target. Therefore, more screening and characterization tests are necessary until the complete establishment of these as effective anti-A. baumannii monoclonal antibodies.

Keywords: Acinetobacter baumannii; monoclonal antibody; immunotherapies 